The University of Wisconsin

Computer Sciences Department

1210 West Dayton Street

Madison, Wisconsin 53706

NATURAL CONVECTION IN AN ENCLOSURF: WITH LOCALIZED HEATING FROM BELOW

by

D. Greenspan and D. Schultz

March 1972

Technical Report \#148 


\title{
NATURAL CONVECTION IN AN ENCLOSURE WITH LOCALIZED HEATING FROM BELOW
}

\author{
by \\ D. Greenspin* and D. Schultat
}

\section{Introduction}

The flow of a gas or of a liquid in a closed cavity is of willosprend interest in fluid mechanics (see, e.9., referencos [1]- $[4],[7],[8]$, md the referencos contained therein). In this paper we will develop a hast, economical numerical method for describing the motion of a fluid in a circulat cylinder with a heat source located centrally on the bottom. The method is a finite difference boundary value technique which will be developed for steady state problems and then adapted to a special class of nonsteady state problems. The entire development extends jn a natural way to any prohlom with an arbitrary axially-symmetric container. Convergence for stoady stite problems has been achieved for Prandtl numbers in tho rango 0.023 to 198.4 .0 and for Grashof numbers in the range 10 to $4(10)^{6}$. Ns applied to time dependent problems, the method is free of roundoff crror amplification, which is inherent in all step-ahead methods.

\section{Problom Statement}

Consider a fluid in a circular cylinder of unit height and of radius h, and assumo that a circular heat source of radius $c$ is contrally loonted in the base.

\footnotetext{
"Computer Sciencos Departmont, University of Wisconsin, Madison, Wisconsin. ${ }^{+}$Mathematies Department, University of Wisconsin, Milwaukee, Wisconsin.
} 
Since any resulting convective flow must be axially symmetric, the motion can be studied as a two dimensional problem. In particular, let us take the mathematical formulation of Torrance and Rockett [8], which, in their terminology, can be summarized as follows.

As shown in Figure 1 , let $O B C D$ be a rectangle with $O B=b, O D=1$. Let $A$ be between $O$ and $B$ with $O A=c$. The problem is to find thrce functions $\psi, \theta$ and $\Omega$ which, inside OBCD, satisfy the partial differential equations

$$
\frac{\partial^{2} \psi}{\partial x^{2}}+\frac{\partial^{2} \psi}{\partial R^{2}}-\frac{1}{R} \frac{\partial \psi}{\partial R}=-R \Omega
$$

(2) $\frac{\partial \partial}{\partial \tau}-\frac{\partial^{2} \theta}{\partial x^{2}}-\frac{1}{R} \frac{\partial \partial}{\partial R}-\frac{\partial^{2} \theta}{\partial R^{2}}+\frac{1}{R}\left(\frac{\partial \psi}{\partial R} \frac{\partial \theta}{\partial x}-\frac{\partial \psi}{\partial x} \frac{\partial \theta}{\partial R}\right)=0$

$$
\begin{gathered}
\frac{\partial \Omega}{\partial \tau}+\frac{1}{R^{2}} \frac{\partial \psi}{\partial x} \Omega+\frac{1}{R}\left[\frac{\partial \psi}{\partial R} \frac{\partial \Omega}{\partial x}-\frac{\partial \psi}{\partial x} \frac{\partial \Omega}{\partial R}\right]+G P^{2} \frac{\partial \theta}{\partial R} \\
-P\left[\frac{\partial^{2} \Omega}{\partial x^{2}}-\frac{1}{R^{2}} \Omega-\frac{1}{R} \frac{\partial \Omega}{\partial R}+\frac{\partial^{2} \Omega}{\partial R^{2}}\right]=0
\end{gathered}
$$

and, in addition, satisty the initial conditions

$$
\psi=\theta=\Omega=0 ; \quad\ulcorner<0,0 \leq x \leq 1,0 \leq R \leq b
$$

and, for $\tau \geq 0$, the boundary conditions

$$
\psi=\frac{\partial \psi}{\partial x}=0 ; 0 \leq R \leq b, x=0
$$




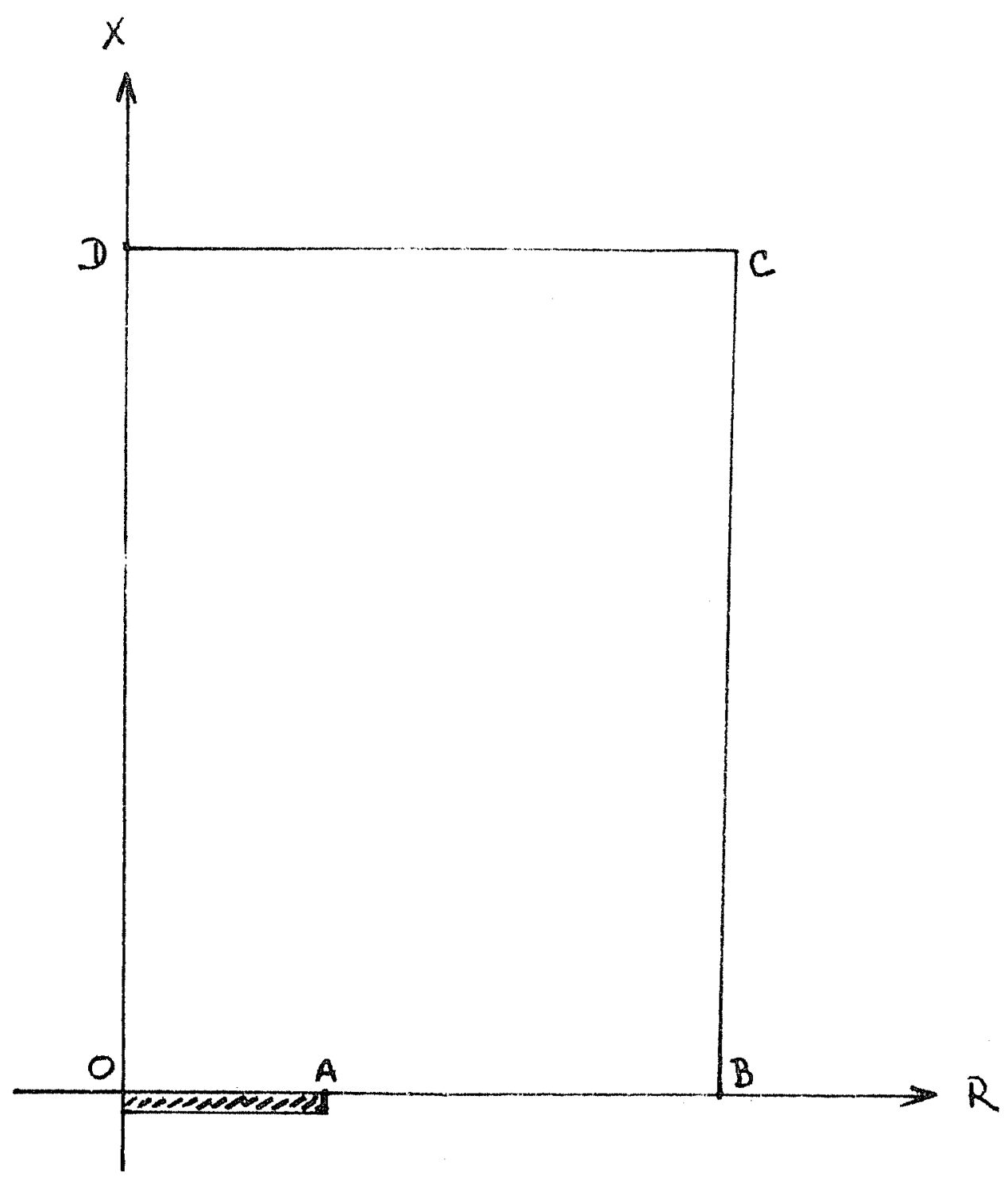

TIGURE; 1 
(6) $\begin{cases}\theta=1 ; & 0 \leq \mathrm{R} \leq \mathrm{c}, \mathrm{x}=0 \\ \theta=0.5 ; & \mathrm{R}=\mathrm{C}, \mathrm{x}=0 \\ \theta=0 ; & \mathrm{c}<\mathrm{R} \leq \mathrm{b}, \mathrm{x}=0\end{cases}$

(7) $\quad \psi=\frac{\partial \psi}{\partial \mathrm{x}}=\theta=0 ; 0 \leq \mathrm{R} \leq \mathrm{b}, \mathrm{x}=1$

(8) $\psi=\Omega=\frac{\partial \theta}{\partial R}=0 ; R=0,0 \leq x \leq 1$

(9) $\psi=\frac{\partial \psi}{\partial \mathrm{R}}=\theta=0 ; \mathrm{R}=\mathrm{b}, 0 \leq \mathrm{x} \leq 1$.

In (1) $-(9), \psi$ is the stream function, $\theta$ is the temperature, $\Omega$ is the vorticity, $\mathrm{P}$ is the Prandtl number, and $\mathrm{G}$ is the Grashof number.

\section{Difference Formulas}

Let us develop, first, the basic difference formulas which will be needed for our numerical method. Throughout, it will be assumed that $h$ and $\Delta \tau$ are positive space and time grid constants, respectively, and that the points $(R, x, \tau),(R+h, x, \tau),(R, x+h, \tau),(R-h, x, \tau),(R, x-h, \tau)$, $(R, x, \tau+\Delta \tau),(R, x, \tau-\Delta \tau)$ are represented by $0,1,2,3,4,5,6$, respectively.

Then, in the usual way [4], the difference approximation for differential Equation (1) is taken to be

$$
\frac{-4 \psi_{0}+\psi_{1}+\psi_{2}+\psi_{3}+\psi_{4}}{h^{2}}-\frac{1}{R} \frac{\psi_{1}-\psi_{3}}{2 h}=-\mathrm{R} \Omega_{0} .
$$


In order to preserve diagonal dominance, equation (2) is differenced in the following special manner ([5], [7]):

$$
\begin{gathered}
\frac{-3 \theta_{6}+4 \theta_{0}-\theta_{5}}{2 \Delta \tau}-\frac{-4 \theta_{0}+\theta_{1}+\theta_{2}+\theta_{3}+\theta_{4}}{h^{2}}-\frac{1}{R} \frac{\theta_{1}-\theta_{3}}{2 h} \\
+\frac{1}{R}\left[\frac{\psi_{1}-\psi_{3}}{2 h} F-\frac{\psi_{2}-\psi_{4}}{2 h} E\right]=0
\end{gathered}
$$

where

$$
\begin{cases}F=\frac{\theta_{0}-\theta_{4}}{h} & \text {, if } \psi_{1}-\psi_{3} \geq 0 \\ F=\frac{\theta_{2}-\theta_{0}}{h} & \text {, if } \psi_{1}-\psi_{3}<0 \\ E=\frac{\theta_{1}-\theta_{0}}{h} & \text {, if } \psi_{2}-\psi_{4} \geq 0 \\ E=\frac{\theta_{0}-\theta_{3}}{h} & \text {, if } \psi_{2}-\psi_{\psi}<0 .\end{cases}
$$

In a similar manner, equation (3) is approximated by

$$
\begin{gathered}
\frac{-3 \Omega_{6}+4 \Omega_{0}-\Omega_{5}}{2 \Delta t}+\frac{1}{\mathrm{R}^{2}} \frac{\psi_{2}-\psi_{4}}{2 \mathrm{~h}} \Omega_{0}+\frac{1}{\mathrm{R}}\left[\frac{\psi_{1}-\psi_{3}}{2 \mathrm{~h}} \mathrm{~F}-\frac{\psi_{2}-\psi_{4}}{2 \mathrm{~h}} \mathrm{E}\right] \\
+G P^{2} \frac{\theta_{1}-\theta_{3}}{2 \mathrm{~h}}-\mathrm{P}\left[\frac{-4 \Omega_{0}+\Omega_{1}+\Omega_{2}+\Omega_{3}+\Omega_{4}}{\mathrm{~h}^{2}}\right. \\
\left.-\frac{1}{\mathrm{R}} \Omega_{0}+\frac{1}{\mathrm{R}} \frac{\Omega_{1}-\Omega_{3}}{2 \mathrm{~h}}\right]=0,
\end{gathered}
$$

where $F$ and $E$ are defined as in (12), but with $\theta$ replaced by $\Omega$. 
Finally, in order to generate $\Omega$ at grid points of either $O B, B C$, or $C D$, at each such point we will use

$$
\Omega=\frac{-2}{h^{2} \mathrm{R}} \tilde{\psi},
$$

where $\tilde{\psi}$ is the value of the stream function at the interior arid point noarest to the given boundary grid point $([4],[7])$.

\section{Numerical Solution of The Stcady State Problem.}

When steady state is physically realizable, it can be doscribed mathematically by (1) - (3) and (5) - (9) morely by dropping tho time derivativos in (2) and (3). We will show how to approximate the solution of such a problem first.

For positive grid size h, construct and number, in the usual way, the set of interior grid points $I_{h}$ and the set of boundary grid points $S_{h}$. (lihough not essential, it will be assumed, for simplicity, that $\frac{1}{h}, \frac{b}{h}$ and $\frac{c}{h}$ aro integers.) The problem then is to approximate $\psi, y$ and $\Omega$ on thoso points of $\mathrm{R}_{\mathrm{h}}+\mathrm{S}_{\mathrm{h}}$ at which they are not already given by (5) - (9).

The procedure is to guess $\psi^{(0)}, d^{(0)}$ and $\Omega^{(0)}$ on these point sets and to procecd rocursively from cach $\psi^{(k)}, j^{(k)}, \Omega^{(k)}$ to ${ }^{(k+1),}(k+1), \Omega^{(k+1)}$ as follows. At each point of $I_{h}$ which is further than $h$ from all points of $\mathrm{S}_{\mathrm{h}}$, writo down (10) with $\Omega_{0}=\Omega_{0}{ }^{(\mathrm{k})}$. Nt each point $(\mathrm{R}, \mathrm{x})$ of $\mathrm{I}_{\mathrm{h}}$ which is 
cxactly a distance $h$ from at least on point $\left(R^{*}, x^{*}\right)$ of $S_{1}$, wrile clown

$$
-4 \frac{y_{i}}{i}+\psi_{j}=0
$$

where $\psi_{i}$ is the value of ${ }^{\prime}$ at $(R, x)$, while $y_{j}$ is the value of at the point $\left(R^{\prime}, x^{\prime}\right)$ which is 2 h from $\left(R^{*}, x^{*}\right)$ and is (ollinoal with $\left(R^{*}, x^{*}\right)$ and $(R, x)$. Inserting the known $\psi$ boundhry values from $(7)-(9)$, one then solvo; the resulting linear algebraic system by successive over-relaxation and denotes the solution by $\bar{\psi}^{(k+1)}$. Then $\psi^{(k+1)}$ is defined by

$$
\psi^{(k+1)}=\gamma^{(k)}+(1-n)^{(k+1)},
$$

where $f$ is a smoothing parameter in the rango $0<1,1$.

To obtain the iterate ${ }^{(k+1)}$, apply (11) without the time dependent term at each interior point of $I_{h}$ and with $\psi_{i}=\psi_{i}(k+1), i=1,2,3,4 . \Lambda \pm s 0$, at each point $(0, x)$ of $S_{h}$, write down

$$
f(0, x)=0(h, x)
$$

Into the system generated by (11) and (17), insert the known values of : given by (6), (7) and (9). Solve the resulting system by successive overrelaxation and denote the solution by $j^{(k+1)}$. Then, $(k+1)$ is defined by

$$
\left.\vartheta^{(k+1)}=\mu \theta^{(k)}+(1-\mu)\right)^{(k+1)}, \quad 0 \leq 11 \leq 1 .
$$

To obtain the iterate $\Omega^{(k+1)}$, first generate $\Omega$ at points of $s_{h}$ by $(14)$, where the vulues of $\psi^{(k+1)}$ are used for $\psi$. Equation (13), without the time 
dependent term, and with $\psi_{i}=\psi_{i}(k+1), i=0,1,2,3,4$, is then applical at each point of $I_{h}$ and the resulting system is solved by successive orerrelaxation. If the solution of this system and the values gonerated by (14) are denoted by $\bar{\Omega}^{(k+1)}$, then $\Omega^{(k+1)}$ is definod by

$$
\Omega^{(k+1)}=\varepsilon \Omega^{(k)}+(1-\varepsilon) \bar{\Omega}^{(k+1)}, 0<i \cdots 1
$$

Finally, the iteration is terminated when, for a given positive tolonnec E, one has uniformly on $\mathrm{R}_{h}+\mathrm{S}_{h}$

$$
\begin{aligned}
& \left|\psi^{(k+1)}-\psi^{(k)}\right|<\varepsilon \\
& \left|y^{(k+1)}-d^{(k)}\right|<\varepsilon \\
& \left|\Omega^{(k+1)}-\Omega^{(k)}\right|<\varepsilon .
\end{aligned}
$$

Using the above algorithm with $\mathrm{b}=1.0$ and $\mathrm{c} \cdots 0.1$, a variety of examples were run. For $\mathrm{P}=0.7$, which was the only parameter choice of Torrance and Rockett, our results are shown, typically, in Pigure 2 and aro in complete agreement with those of Torrance of Rockett. Howevel, in adplition, our method was also applied to the two extreme cases $P=0.023$, which corresponds to liquid mercury, and to $\mathrm{P}=9884.0$, which corresponds to aircraft engine oil, and typical results are shown in l'igure 3 . For $\Gamma=0.023$ the maximum value of $\psi$ varies from 0.0001 when $G=1000$ to $: 0.019$ when $G=50000$, while for $P=9884$ the maximum of $y$ is 3.0 for $G=20$. 

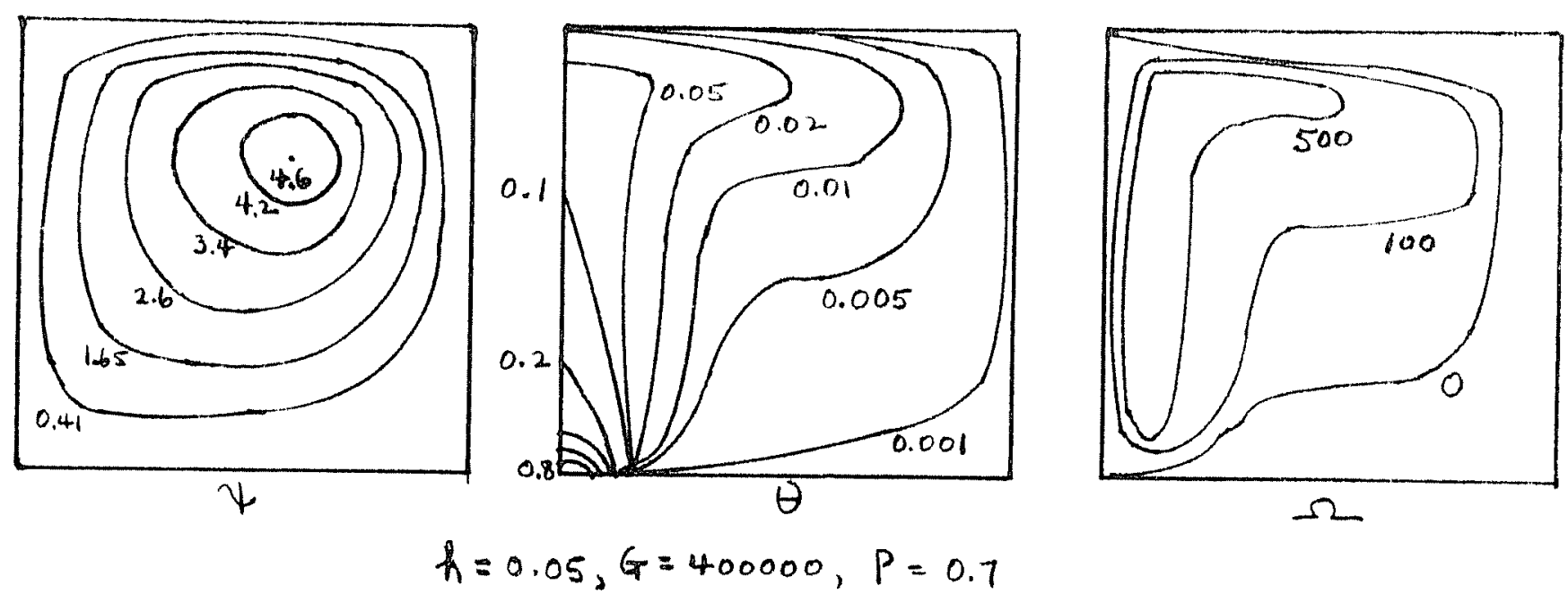

$$
h=0.05, G=400000, P=0.7
$$
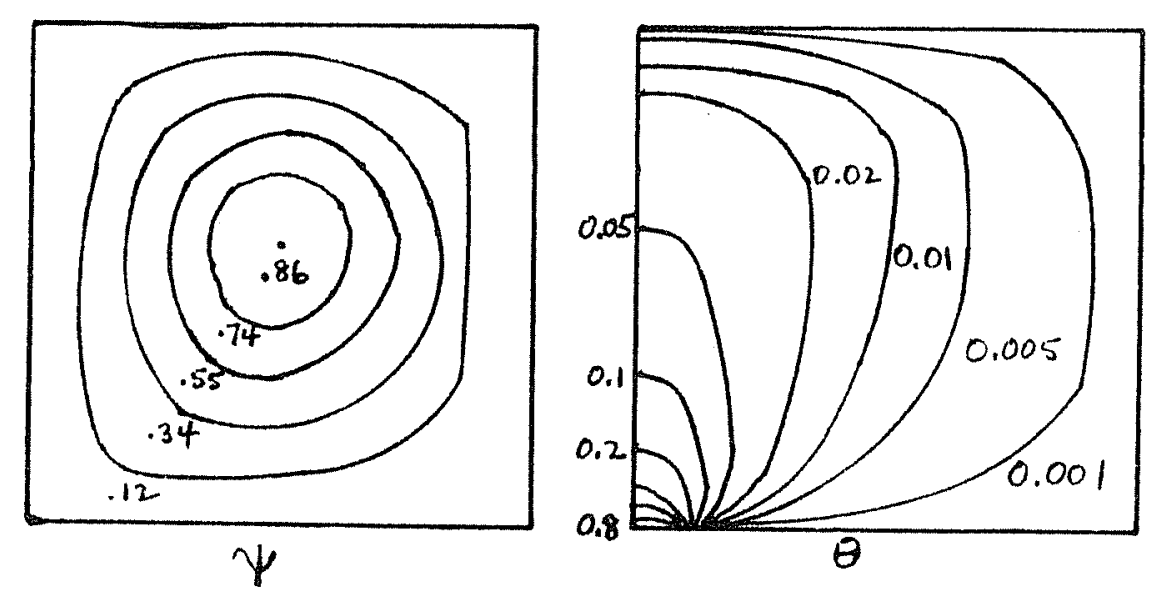

$$
h=0.05, G=40000, P=0.7
$$
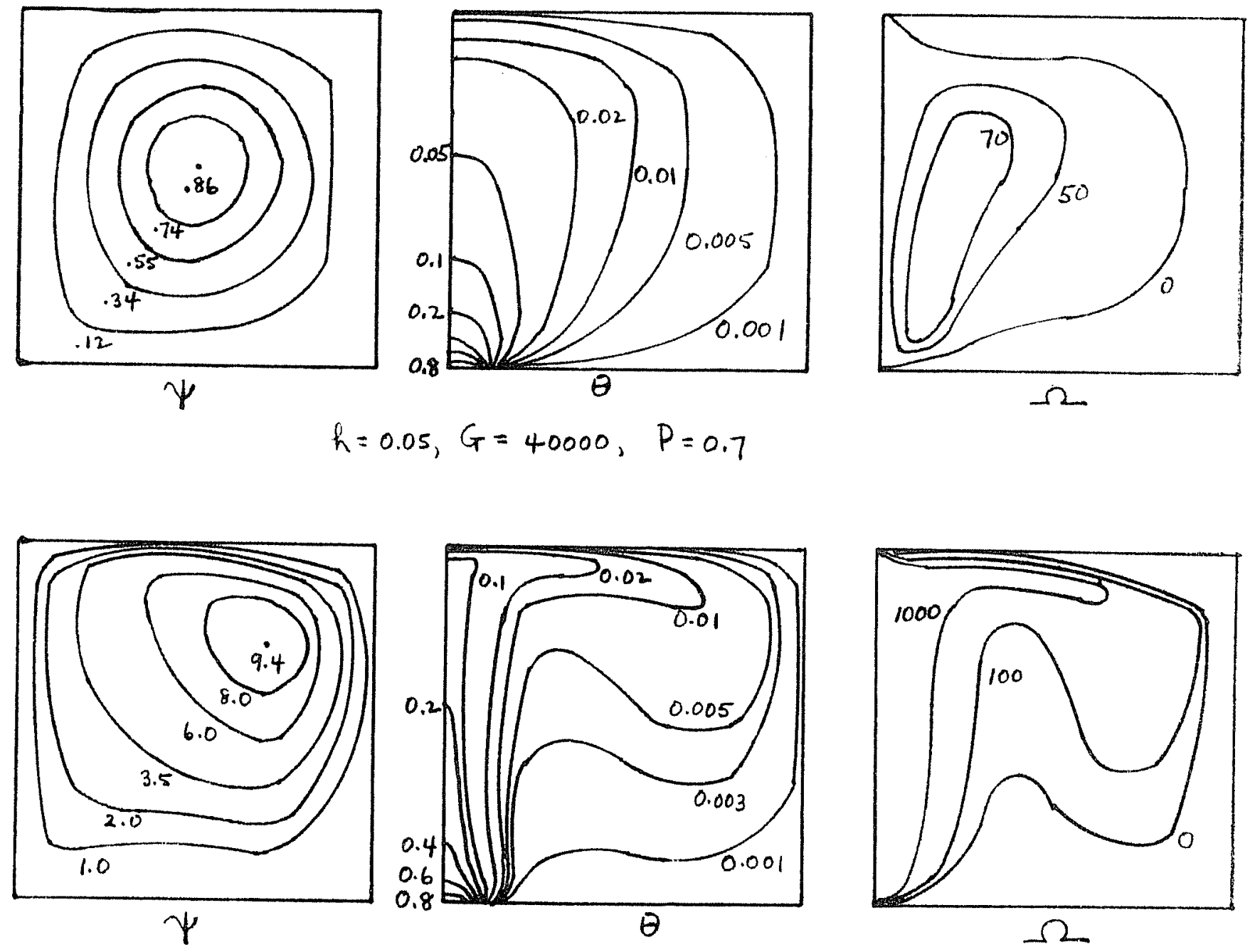

$$
h=0.02, G=4000000, P=0.7
$$



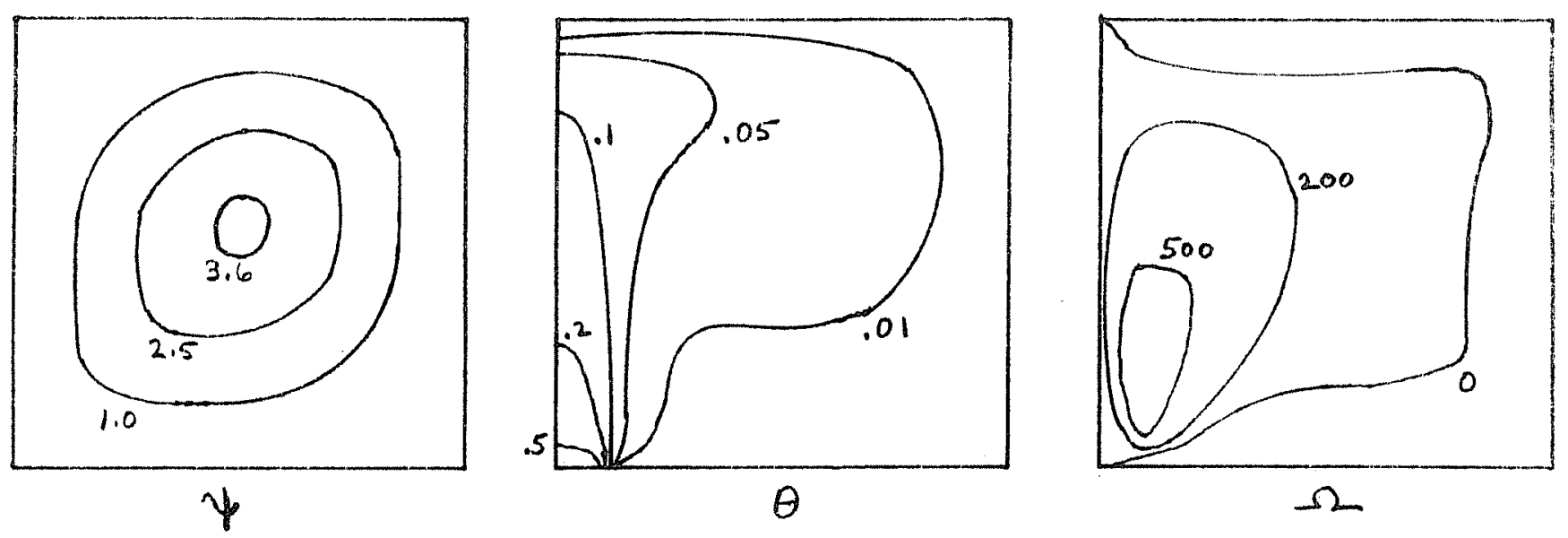

$h=0.05, G=10, P=9884$
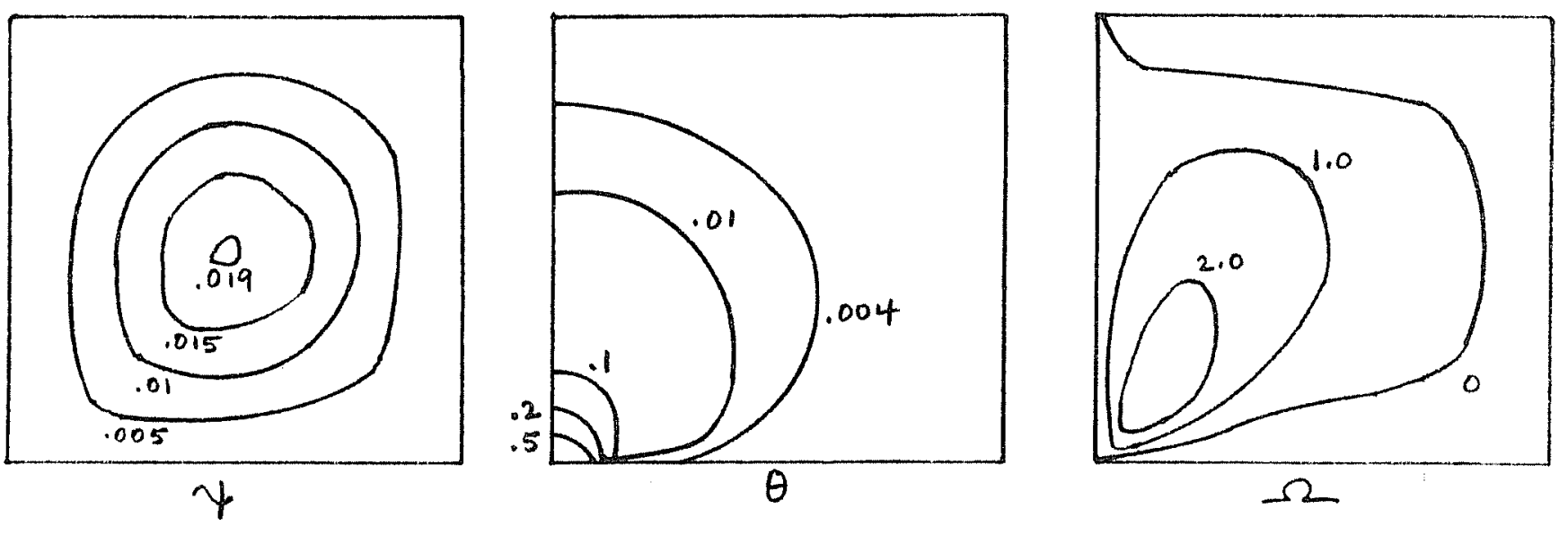

$h=0.05, G=50000, P=0.023$

FIGURE 3 
To illustrate the speed of the method, the threc cascis $h=0.1,6=40000$; $h=0.1, G=400000 ;$ and $h=0.05, G=40000$, with $P=0.7$ ame $:=10^{-6}$ required a total computing time of two minutes and scven soconds on the UNIVAC: 1108.

With regard to convergence, it is of interest to note that, as observed previously $([4],[5],[7])$, any choice of $p, 1$, or 6 outside the rangos $0=0=0.4,0<\mu \leq 0.4,0.6 \leq 6<1$ resulted, invariably, in divegont behavior. In addition, a method analogous to that doscribod hor, but in which the natural sclection of central difference formulas was mate for all derivatives in (2) and (3), was explored and, though the rosults agreod with those already obtained, the method was of limited viability due to the loss of diagonal dominance.

\section{Numerical Solution of the Time Dependent Problem.}

Time dependent problems are solved usually by initial valu techmifus: Such methods often suffer from numerical cror accumulation with the advance in time and from severe stability limitations when the dynamical equations are nonlinear. Since boundary value techniques are not prone to these purticular shortcomings, let us show how to extend the method of Section $t$ to initial value problems by first converting the ajven problom (1) - (9) to a houndary value problem. In particular, this can be done readily if (1) - (9) has a stoady state solution $\psi(R, x, \cdots), U(R, x, \cdots), n(R, x, \infty)$ which is 
independent of the initial conditions. Under the assumption that such a solution exists, it is prescribed at $c=\infty$ and condition (4) is (roppod.

The numerical method can be formulated now as follows ([5], [7]).

Solve the steady state problem numerically as described jn section 4. Denote the numerical solution by

$$
\psi^{(k+1)}=\psi(R, x, 0), v^{(k+1)}=(R, x, \infty), \Omega^{(k+1)}=\Omega(R, x, \cdots) .
$$

Next, fix a value $r=: T$ and define $\psi(R, x, T),:(R, x, T), \Omega(R, x, T)$ such

that at the points $(b-n h, 1-m h, T), n=1,2, \ldots, \frac{b-1}{h}, m=1,2, \ldots, \frac{2}{h}$, one has

(21) $\psi(R, x, T)=\psi(R, x, m), \forall(R, x, T)=(R, x,(x), \Omega(R, x, T)=\Omega(R, x, n)$.

Consider then the boundary value problem defined by (1) - (3), (5) - (9) and $(21)$ on the rectangular parallelopiped defincd by $0 \leq k-1,0 \cdots x-1$, $0=r \leqslant \mathrm{~T}$. Apply the techniques described in Section to this throe dimensional problem by cmploying the fullequations (11) and (13) with space time step $h$ and time grid step $\Delta l$, thus yiclding the numerical solution.

Using the above method with $b=1.0$ and $c=0.1$, a varicty of exmples again were run efficiently and economically. Typical problems and results are shown in Figures $4-6$. Interestingly enough, the numerical results themsalves s]ways indicated whether or not a choice of I' was either too small or too large, for, when $T$ was too small, the numerical solution 
showed an erratic change only between $T-\Lambda t$ and 'T', whereas, when 'l' was too large, there would be no change in the numerical solution for many time steps preceding $T$. Running times usually varied between 3 and 20 minutes with $A t$ in the range from 0.001 to .025 and $T$ in the range from 0.007 to 2.0 . One could use such relatively small values of $\mathrm{T}$ because, both mathematically and physically, steady state, when it exists, is usually reached rapidly. 
$\psi$
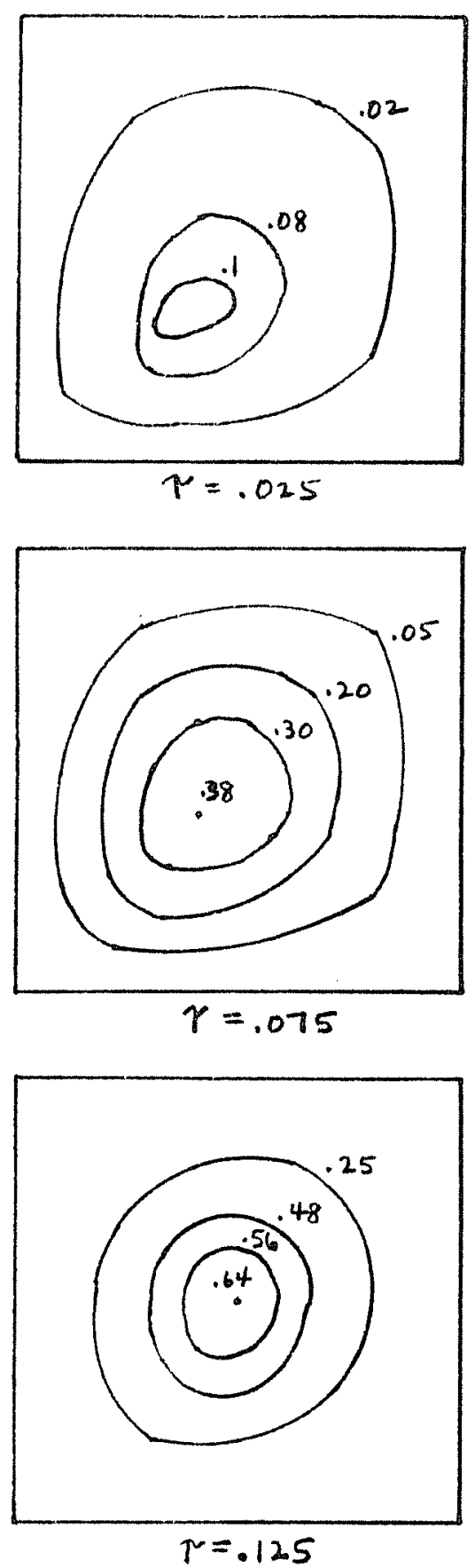

$\tau=.125$
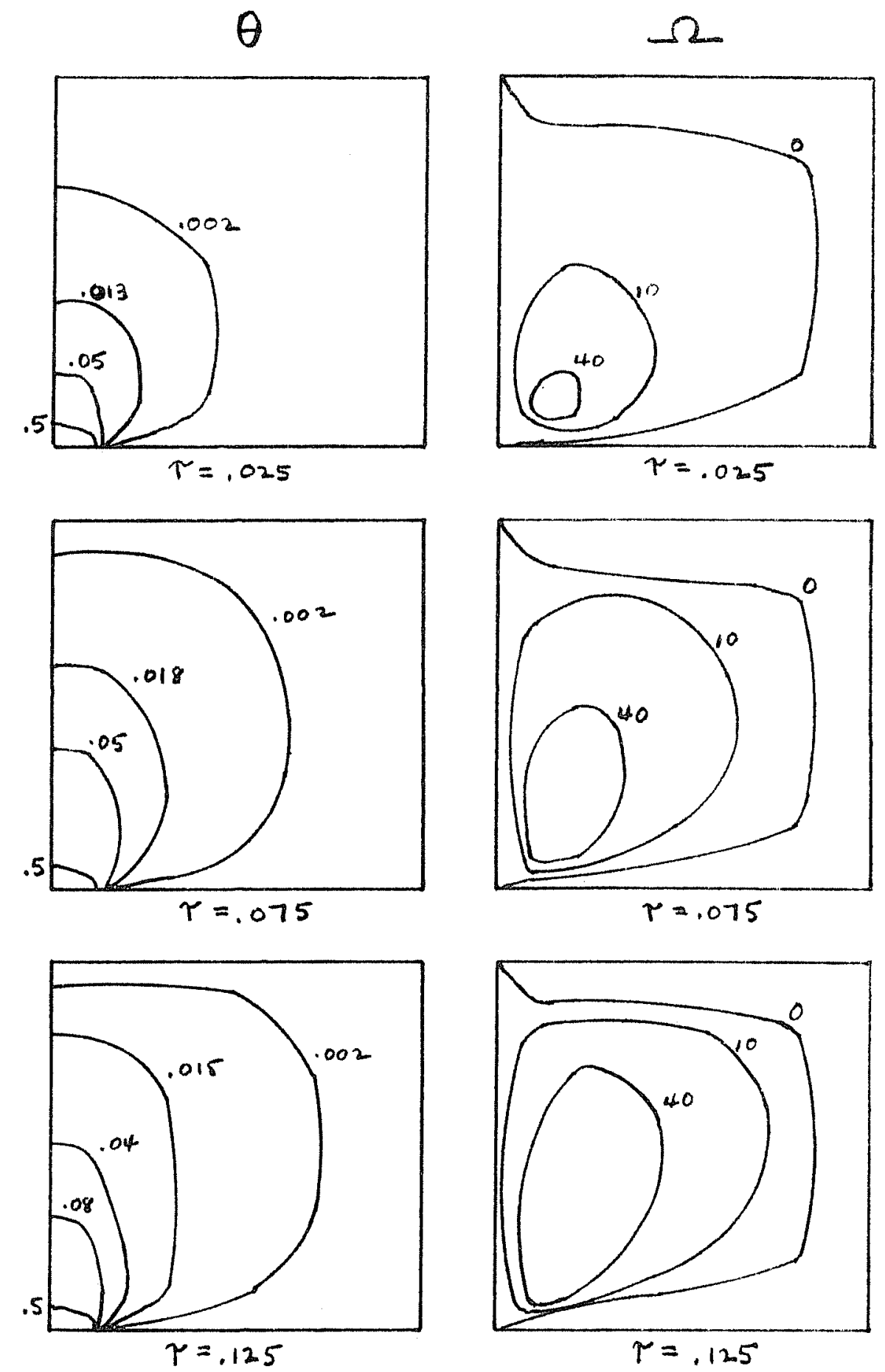

FIGURE 4. $\mathrm{h}=0.1, \wedge \mathrm{l}=0.001, \mathrm{G}=40000, \mathrm{P}=0.7$ 

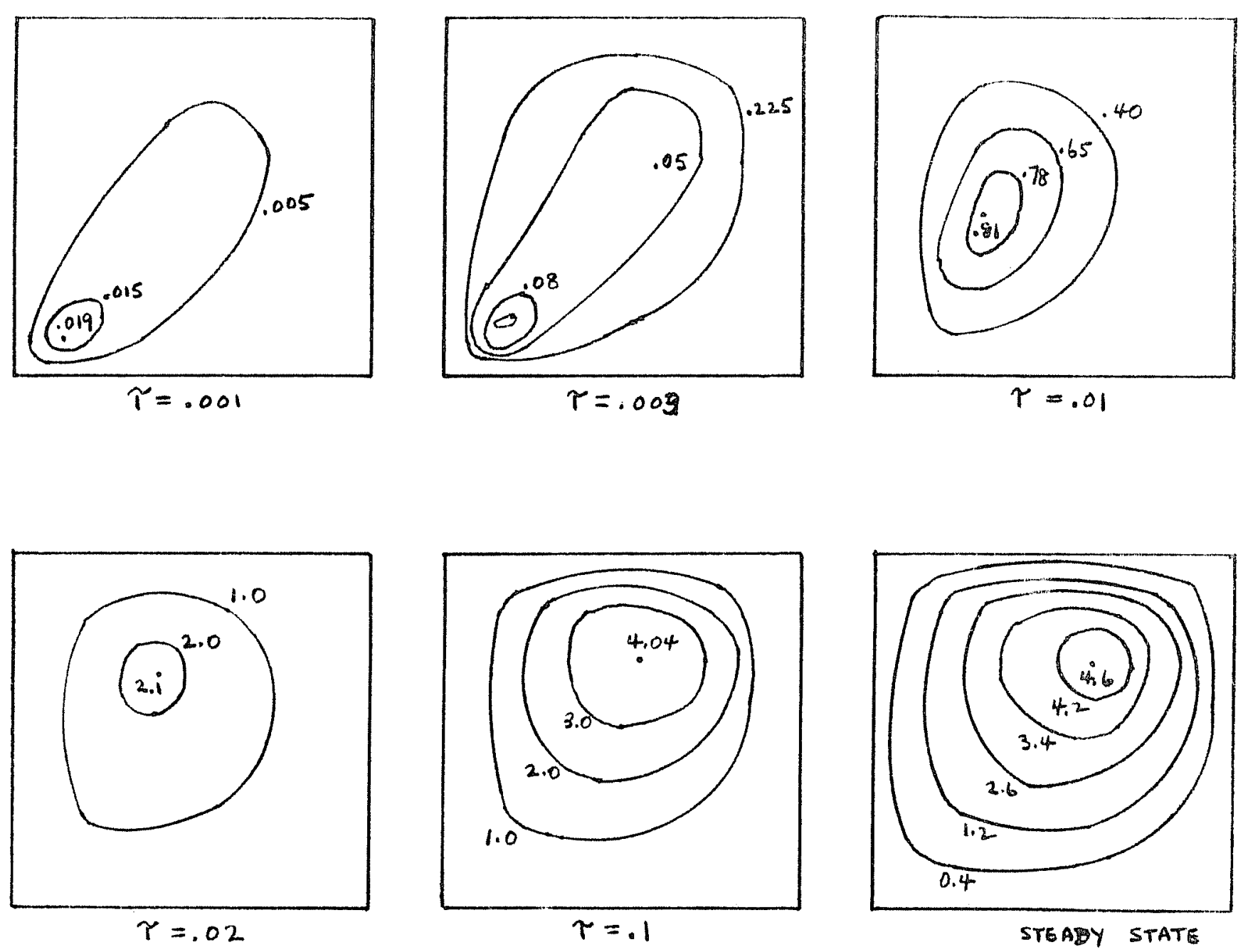

FIGURE 5. $\psi$-curves, $h=0.05, \wedge r=0.001, G=400000, P=0.7$ 

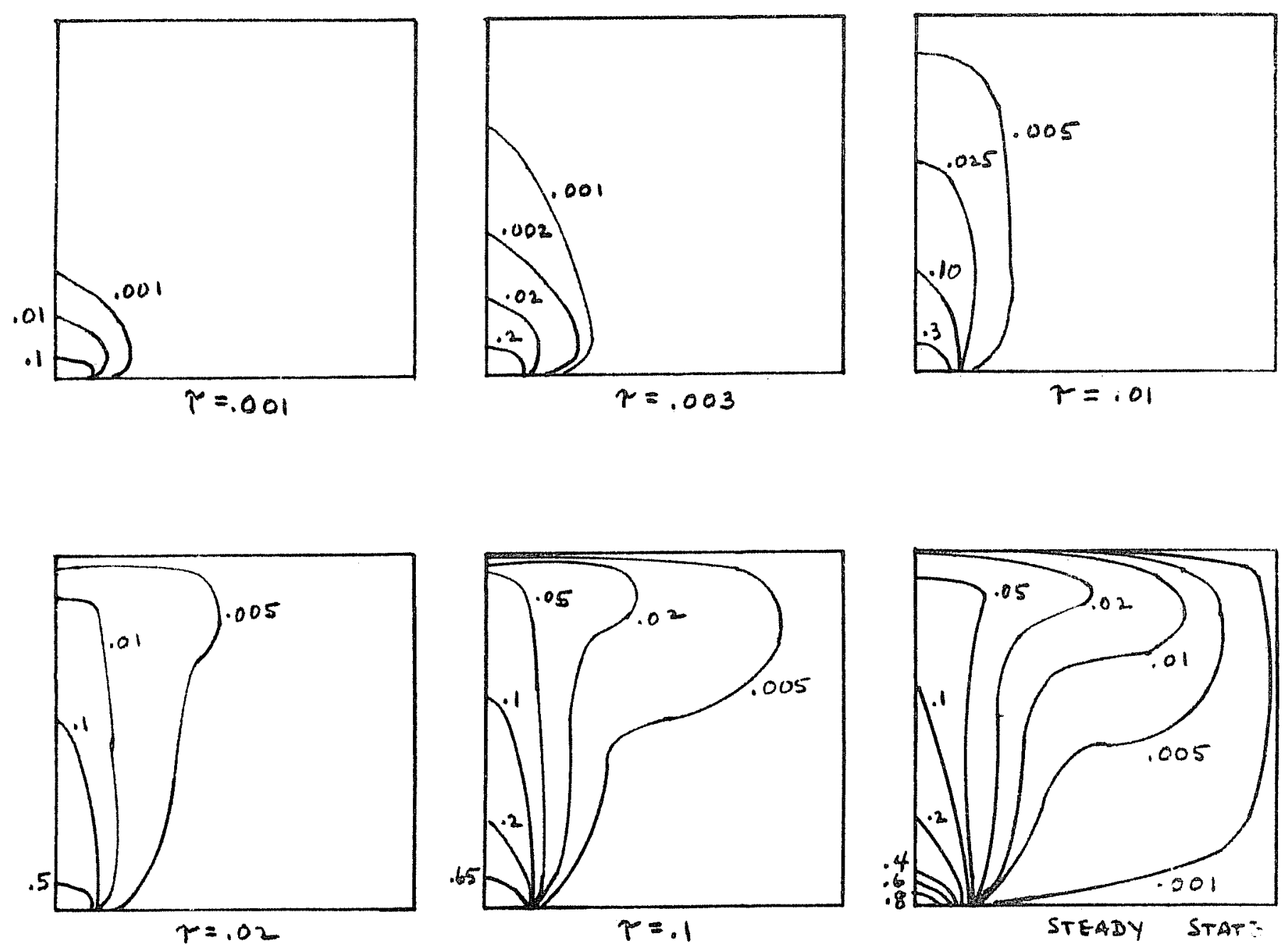

FIGURE 6. $\theta$-curves, $h=0.05, \Lambda T=0.025, G=400000, P=0.7$ 


\section{References}

1. H. Z. Barakat and ]. A. Clark, "Analytical and exporimontinl atuly of the transient laminar natural convection flows in partially filled liquid containers," Proc. Third Int. Heat Trans. Conf., vol. 2, Nmor. Inst. Chem. Fing., Chicago, Ill., 1966, pp. 150-162.

2. G.K. Batchelor, "Heat transfer by free convection across a closud cavity between vertical boundaries at difforent temporaturos, "Qlkart. Appl. Math., 2, 1954, pp. 209-233.

3. J. Fromm, "The time dependent flow of an incompressible viscous fluid", Methods in Computational Physics, vol. 3, Mcadomic Pross, N. Y., 1964, pp. $346-382$.

4. D. Greenspan, "Numerical studies of prototype cavity flow problems", Computer Jour. , 12, 1969, pp. 89-94.

5. , "Numerical solution of a class of nonsteady cavity llow problems," BIT, 8, 1968, pp. 287-294.

6. _Numerical studies of steady, viscous, incomprossiblo flow in a channel with a step," Jour. Eng. Math., 3, 1969, pp. 21-28.

7. D. H. Schultz, Experimental Numerical Solution of tho Navier-Stokes Equations for the Flow of a Fluid in a Hoated Closed Cavity, Ph. D. Ihesis, University of Wisconsin, Madison, 1970, to appoar in publishod Torm in OJMAM. 
8. K. E. Torrance and J. A. Rockett, "Numerical study of natural convection in an enclosure with localized heating from below - creeping flow to the onset of laminar instability," Jour. Fluid Mech., 36, 1969, pp. $33-54$. 